\title{
Titel/Title: Ordoliberalismus als ökonomische Ordnungstheologie
}

Autor*innen/Author(s): Philip Manow

Veröffentlichungsversion/Published version: Postprint

Zeitschriftenartikel/Journal article

\section{Empfohlene Zitierung/Recommended citation:}

Manow, Philip (2001): Ordoliberalismus als ökonomische Ordnungstheologie Ordoliberalismus als ökonomische Ordnungstheologie. In: Leviathan, 29, in: Leviathan, 29, $179-198$.

Verfügbar unter/Available at:

(wenn vorhanden, bitte den DOI angeben/please provide the DOI if available)

https://doi.org/10.1007/s11578-001-0012-z

Zusätzliche Informationen/Additional information:

Der Autor kann kontaktiert werden unter:

manow@uni-bremen.de 


\section{Philip Manow}

Ordoliberalismus als ökonomische Ordnungstheologie ${ }^{1}$

Für Gerhard Lehmbruch

\section{Ordoliberaler Antiliberalismus}

Ich möchte mit einem etwas längeren Zitat beginnen:

„Das wichtigste Erfordernis jeder Wirtschaftsordnung, die diesen Namen verdient, ist, daß die politische Führung Herr der Gesamtwirtschaft im ganzen wie in ihren Teilen sein muß; es ist notwendig, daß die staatliche Wirtschaftspolitik das wirtschaftliche Geschehen geistig und machtmäßig in den Griff bekommt. Dies ist nur möglich, wenn die Wirtschaft durchsichtig und streng geordnet ist und wenn diese Ordnung, die eine rechtliche und politische Ordnung ist, vom Staat mit sachlichem Verständnis gehandhabt, von der Nation geistig erfaßt und erlebt und von den wirtschaftenden Volksgenossen mit Hingabe und Disziplin befolgt wird. Für die Ordnungsmittel und die Führungsbefehle gilt daher der Satz, daß sie sachlich geeignet sein müssen, eine solche Ordnung zu erzeugen, und daß sie ferner stark genug sein müssen, damit es der politischen Führung ermöglicht wird, ihre Herrschaft über die Wirtschaft zu behaupten."

Wenn man dieses Zitat datieren und einem Autor zuschreiben möchte, so bietet die Rede vom 'wirtschaftenden Volksgenossen' eine erste Handhabe. Und richtig, der Text stammt aus dem Jahr 1937. Wenn man aber weiterhin meint, dass die Rede von den 'Führungsbefehlen' und 'Ordnungsmitteln', von der 'politischen Herrschaft über die Wirtschaft', von Strenge, Stärke und Disziplin auf einen nationalsozialistischen Proponenten einer 'gebundenen Wirtschaft' hinweist, so liegt man grundfalsch. Der Autor ist einer der Säulenheiligen des Ordoliberalismus: Franz Böhm. Das Zitat ist entnommen der programmatischen Schrift „Die Ordnung der Wirtschaft als geschichtliche Aufgabe und rechtsschöpferische Leistung", Heft 1 der von Böhm, Walter Eucken und Hans

\footnotetext{
${ }^{1}$ Friedrich Wilhelm Graf war es, der mich auf die protestantischen Wurzeln des Ordolibera- lismus aufmerksam gemacht und mich darin bestärkt hat, der 'religiösen Tiefengrammatik' dieser Wirtschaftsdoktrin nachzugehen. Gerhard Lehmbruch hat mich durch detaillierte Kritik an einer frühen Version gezwungen, meine Thesen viel genauer zu formulieren. Ich bin beiden zu großem Dank verpflichtet. Weiterer Dank geht an Jens Alber, Bodo von Greiff, Philipp Gensehei und Wolfgang Seibel.
} 
Grossmann-Doerth herausgegebenen Schriftenreihe „Ordnung der Wirtschaft", die den Ordoliberalen bis heute als authentischer Ort der intellektuellen Grundlegung der neuen wirtschaftspolitischen Doktrin gilt (Zitat von S. 10). $(\leftarrow$ S. 179)

Nun ist es weder so, dass man viel Ausdauer besitzen muss, um in dem reichen ordoliberalen Schrifttum vereinzelte 'inkriminierende' Stellen vergleichbaren Inhalts zu finden. Die genannte Schrift über 'Die Ordnung der Wirtschaft', wie auch viele andere ordoliberale Abhandlungen aus dieser und späterer Zeit, sind voll von autoritären und 'volkserzieherischen' Bekundungen, so dass es sich nicht nur um vereinzelte, bloß semantische Konzessionen an die neuen Machthaber handelt, denen man doch sonst angeblich so reserviert bis schließlich feindselig gegenüberstand. Noch ist der Text im Zuge einer frühen Euphorie über das Ende der politischen und wirtschaftlichen Agonie Weimars verfasst worden, sondern im Jahr 1937, als die Ernüchterung über die Nazis und ihre Politik im bürgerlichen Lager schon weit verbreitet war. Der Text liest sich denn auch eher wie eine an das Regime selbst gerichtete Mahnung, endlich von dem polykratischen Hin-und-Her in der Wirtschaftspolitik zu lassen und stattdessen einen autoritären Ordnungsentwurf 'aus einem Guss' zu verwirklichen (vgl. Herbst 1982).

Aus dem obigen Zitat (und aus ähnlichen Äußerungen der 1930er Jahre von Eucken, Böhm, Rüstow, Röpke, von Dietze, Müller-Armack, oder Lampe) spricht aber natürlich auch keine Nazigesinnung. Doch eine liberale Gesinnung wird man diesem Lobgesang auf die ,politische Verfassung des nationalen Wirtschaftslebens" und auf die ergänzend für nötig erachtete ,gewaltige nationale Erziehungsarbeit" wohl auch kaum nachsagen können (Böhm 1937, S. 53 und 51). Böhms Vorstellung von einer gesellschaftlichen „Kompositionsidee", einer „Partitur des sozialen und politischen Lebens" (Böhm 1937, S. 11), die nur von der Rechts- und Wirtschaftswissenschaft zu identifizieren sei, damit dann der Staat eine ihr entsprechende „geschlossene und straffe" Ordnung der Wirtschaft erschaffe sowie eine ,mit dem Bewegungsprinzip in Einklang stehende soziale Gliederung" (ebd., S. 52), fällt hinsichtlich ihrer 'Anmaßung von Wissen' (Hayek) nur wenig hinter andere Utopien totaler Gesellschaftsgestaltung zurück.

Nun bereitet es immer Schwierigkeiten, sich auf eine verbindliche Definition zu einigen, was man unter 'liberal' und 'Liberalismus' verstehen möchte. Eine Einigung darüber, welche Einstellungen und Positionen nicht mit einer liberalen Position vereinbar sind, fällt hingegen oft leichter. Folgen wir Stephen Holmes ,preliminary checklist of antiliberalism's distinguishing characteristics" (Holmes 1993, S. 5), so zeichnet sich Anti-Liberalismus aus durch eine ausgesprochene Krisenrhetorik (Antiliberale treten dabei selbst als ,doctors of disorder" auf; ebd., S. 5), 
- durch ätzende Zivilisationskritik und lautes Klagen über den moralischen Werteverfall, $(\leftarrow \mathbf{S}$. 180)

- hervorgerufen durch Materialismus, Atomismus, Vermassung und Verstädterung und der damit einher gehenden Wurzel- und Bindungslosigkeit des modernen Menschen, hervorgerufen ebenso durch die Abkehr von der christlich-abendländischen Wertetradition (Röpke: „religiöser Reserveverzehr"; Müller-Armack: „Glaubensabbau"; Eucken: „Zerstörung der Werte");

- an ihre Stelle sei blanke Ichsucht, egoistischer Konsumismus und ein ober- flächlicher Szientismus und Fortschrittsglaube getreten.

$\mathrm{Zu}$ dieser anti-liberalen Zivilisationskritik gesellen sich oft

- die Idealisierung vergangener, irgendwie glücklicherer Zeiten (im wirtschaftli- chen Kontext das Idealbild einer handwerklich-agrarisch geprägten Gesellschaft),

- ein markanter Anti-Pluralismus, tiefsitzende Vorurteile gegenüber dem parteipolitischen Interessenausgleich und ein erstaunlich vorbehaltsloses Vertrauen in die hierarchische Steuerung durch den Staat,

- schließlich ein bedenkenloses Rekurieren auf einen substanziellen Gemeinwohl-Begriff, der gegen die gesellschaftlichen Partikularinteressen in Anschlag gebracht wird.

Nach dieser 'checklist' ist eine Zuordnung des Ordoliberalismus zum Antiliberalismus schwer zu bestreiten: Rüstows Klagen über die Vermassung als 'Elephantiasis' der modernen Zeit, Röpkes Horror vor der Moralzersetzung in den modernen 'Riesenstädten', Euckens prononcierter AntiPluralismus und Ruf nach dem starken Staat, der „,die Kraft findet, sich von dem Einfluß der Massen frei zu machen" (1932, S. 318), die Polemik der Ordoliberalen gegen den westlichen Parlamentarismus als Veranstaltung zur 'rücksichtslosen Ausbeutung des Staates durch die Interessenhaufen' (Röpke), Röpkes, Rüstows ('Vitalpolitik') und Erhards romantisierende Vorstellung einer agrarisch-handwerklich geprägten Wirtschaftsordnung, in der ,das Bauerntum [...] als stärkste[r] Schutzwall gegen den Bolschewismus" fungieren sollte (Rüstow 1963, S. 73), dazu Böhms Lobgesang auf die autoritäre Staatssteuerung etc. pp. - ein spezifisch liberales Element ist in diesen Positionen beim besten Willen nicht zu entdecken.

Betrachtet man die frühe formative Phase der neuen ordoliberalen Denkschule am Ende der 1920er, Anfang der 1930er Jahre, so fällt zunächst ihre Nähe zur konservativen Revolution auf. Euckens 
„Strukturwandel des Staates und Krise des Kapitalismus" (1932) und Rüstows Rede vor dem Verein für Socialpolitik, ,Interessenpolitik oder Staatspolitik?" (1932 [1986]), bis heute als Gründungsmanifeste des Ordoliberalismus gefeiert, sind militante Abrechnungen mit dem 'Weimarer System', mit den 'Weimarer Systemparteien', mit dem verhassten „Pluralismus" (Rüstow 1932, S. 255), unter dem man sich nichts anderes vorstellen konnte als die Strategie, den „Staat als Beute" zu betrachten (ebd.). Die tiefsitzenden Ressentiments gegen den Weimarer Parlamentarismus brechen sich unter den Ordoliberalen kaum anders Bahn als zur gleichen Zeit in den Reihen der konservativen Revolution. $\mathrm{Ob}$ als Abscheu vor dem widerwärtigen 'Fraktionenkrakeel', ob als $(\leftarrow$ S. 181) Polemik gegen die „Vermassung, Parteiung, Sprachenverwirrung, Auflösung und Gruppenfehde" in der modernen Demokratie (Böhm 1937, S. 47), ob als Klage über die politische „Versumpfung des Kapitalismus" durch „den gewachsenen politischen Einfluß der Massen" (Eucken 1932, S. 315), ob als Ruf nach „Autorität und Führertum" (Rüstow 1932 [1986), S. 70) - die Ordoliberalen unterscheiden sich zunächst allenfalls in sprachlichen Nuancen von den konservativen Revolutionären (vgl. Sontheimer 1968). Und auch die von den Ordoliberalen empfohlene Therapie gegen die wirtschaftliche und politische Agonie Weimars befindet sich in weitgehender Übereinstimmung mit dem Programm der Neuen Rechten, wie es zur gleichen Zeit von Edgar Jung oder Walther Schotte formuliert wurde. So fällt es schwer, zwischen dem Ruf Walther Schottes (dem Vordenker des von Papen-Kabinetts) nach einem „,neuen Staat, der ein starker Staat sein muß, frei von Interessen, gerecht in sich, unabhängig von den Parteien" (zitiert nach Sontheimer 1968, S. 161) und Alexander Rüstows zur gleichen Zeit artikuliertem Sehnen nach dem „starken Staat, [...] der über den Gruppen, über den Interessenten steht" (Rüstow 1932 [1986), S. 69-70), einen substanziellen Unterschied festzustellen.

Dieser antiliberale Grundzug des Ordoliberalismus ist natürlich seit längerem bekannt, auch wenn man in der ordoliberalen Hagiographie bis heute hartnäckig versucht, ihn zu ignorieren. Schon 1932 sah sich Hermann Heller angesichts der antiliberalen Grundströmung dieser bürgerlichen Wirtschafts'liberalen' dazu ver- anlasst, vom autoritären Liberalismus zu sprechen (Heller 1932). Und die Arbeiten von Krohn (1981), Herbst (1982), Abelshauser (1984) oder Haselbach (1991) bestätigten Hermann Hellers Diagnose im Wesentlichen. Die von Rüstow u.a. selbst gewählten Begriffsschöpfungen wie 'neuer', 'radikaler', 'konservativer' oder 'rabiater Liberalismus' bzw. „,liberaler lnterventionismus" (Rüstow 1932 [1986), S. 67) zeigen, dass auch den Vertretern der neuen Doktrin durchaus bewusst war, dass sie nicht auf ausgetretenen liberalen Pfaden wandelten. Ludwig Erhard formulierte 1942 eine die Ordoliberalen einigende Einsicht, wenn er lakonisch feststellte: „Das liberale Prinzip hat versagt" (zitiert nach Hentschel 1996, S. 26). Für den klassischen Liberalismus hatten auch die Ordoliberalen nichts als Verachtung übrig. Rüstow denunzierte ihn 
gerne als Steinzeit- oder „Paläoliberalismus" (s. etwa Rüstow 1960).

Doch werden wir den ordoliberalen Denkern nicht gerecht, wenn wir sie le- diglich als minder radikale Fraktion innerhalb des neukonservativen Überdrusses am System von Weimar verstehen. Dass die Wurzeln des Ordoliberalismus nicht rückstandslos auf den zeitgenössischen Kontext der konservativen Revolution zurück geführt werden können, zeigt sich insbesondere darin, dass (etwa ab 1938) die Ordoliberalen sich schließlich schroff vom Nazi-Regime abwenden - bis hin zur aktiven Teilnahme an den Planungen für die Nachkriegsordnung nach dem nun auch von ihnen für unvermeidlich gehaltenen Untergang des Regimes (Blumenberg-Lampe 1973). Hierauf gründet der für den Erfolg dieses wirtschaftspolitischen $(\leftarrow$ S. 182) Konzepts so wichtige Nachkriegs-Mythos, die Ordoliberalen hätten von Anfang an und prinzipiell, nämlich aus liberaler Grundüberzeugung, den neuen Machthabern reserviert bis feindlich gegenüber gestanden.

Wie passt das zusammen? In einer stimmigen Erklärung des deutlich komplizierteren Karriereverlaufs des Ordoliberalismus wären also sowohl die zunächst stark autoritäre Färbung des Ordoliberalismus, der spätere Bruch mit dem Nazi-Regime sowie der bis weit in die Nachkriegszeit hinein reichende ordoliberale Antiliberalismus plausibel zu machen. Eine solche Erklärung biete ich hier an, indem ich den religiösen Wurzeln dieser Wirtschaftsdoktrin nachgehe.

\section{Die Wiedergeburt protestantischer Sozialreform aus dem Geist der Wohlfahrtsstaatskritik}

Der Ordoliberalismus entsteht in der zweiten Hälfte der 1920er Jahre als Reaktion auf Inflation und Weltwirtschaftskrise. Der eigentliche Grund der Krise Weimars ist jedoch in den Augen dieser Autoren nicht wirtschaftlicher, sondern politischer Natur. Der Ordoliberalismus ist die wirtschaftspolitische Doktrin eines sich radikalisierenden, tiefgreifend „statusverunsicherten" Bürgertums (Mommsen 1987, S. 300), das sich in der Weimarer Republik zunehmend wirtschaftlich aufgerieben sah zwischen den korporatistischen Großgruppen von Arbeit und Kapital, sich als politisch marginalisiert empfand, das sich aber schließlich auch konfessionell in die Enge getrieben fühlte. Träger der Doktrin ist eine bürgerlich-protestantische Bildungselite, die den Weimarer Korporatismus als handfeste ökonomische Be- drohung empfand und zugleich als Herausforderung eines konfessionellen Hegemonieanspruchs, den der lutherische Protestantismus seit 1871 für sich beansprucht hatte.

Wichtiges Element der bürgerlichen Kritik am System von Weimar war der immer heftiger werdende Angriff auf den Weimarer Wohlfahrtsstaat. Auch diese Polemik hatte eine sozio-ökonomische, politische und konfessionelle Dimension. Denn von dem kräftig expandierenden Wohlfahrtsstaat 
schienen vor allem die christlichen (katholischen) und freien (sozialistischen) Gewerkschaften, entspre- chend parteipolitisch das Zentrum und die Sozialdemokratie zu profitieren. Die Gewerkschaften waren gut in die Selbstverwaltung des Bismarckschen Sozialstaates integriert, während der soziale Protestantismus ein heterogenes, aber bei aller Heterogenität doch durchgängig (bildungs-)bürgerliches Projekt blieb. Ohne Mobilisierungserfolg in den Arbeiterschichten fehlte ihm die organisatorische Verbindung mit dem besonders auf die Interessenlage der Arbeitsschichten ausgerichteten deutschen Wohlfahrtsstaat. Nur in der Fürsorge, einem eher nachrangigen Betätigungsfeld im deutschen Sozialversicherungsstaat, auf die zudem in der Weltwirt- schaftskrise zunehmend alle sozialpolitische Anpassungslast abgeladen wurde, $(\leftarrow$ S. 183) konnte der soziale Protestantismus ein genuines Betätigungsfeld finden (s. vom Bruch 1985).

Der Wohlfahrtspaternalismus der bürgerlich-protestantischen Sozialreformer, so schien es, hatte sich in der modernen Gruppendemokratie zunehmend leer gelaufen. Der bürgerliche Sozialreformer Heinz W. Marr schrieb pointiert: „Unsere Sozialpolitik rechnete mit dem Staat preussischen Stils. Von woher wollen wir jetzt eingreifen [..] und wer vollzieht nun die 'Korrektur der Wirtschaft durch die Sittlichkeit'?" (zitiert nach Janssen 1998, S. 223). In der Tat, die ursprünglich protestantische Sozialreform schien nun 'konfessionell enteignet'. So wechselte die vormalige 'Stoßtruppe der Sozialreform' ins Lager der Wohlfahrtsstaatskritik. Sozialpolitik, nicht mehr getragen von einer elitären Modernisierungskoalition aus Staatsbürokratie und (protestantischem) Bildungsbürgertum, sondern nun Gegenstand des Parteien-Überbietungswettkampfs, und hier besonders vorangetrieben von der Groß-Koalition aus Zentrum und Sozialdemokratie, den Anti-Protestanten und vormaligen Reichsfeinden, wird nun zum Objekt der bürgerlichen Polemik, die schnell das gesamte politische System Weimars ins Visier nimmt.

Der Ordoliberalismus nimmt von dieser Polemik seinen Ausgang, wobei die konfessionellen Tone im Klagen über die politische Marginalisierung des liberal-protestantischen Milieus nicht zu überhören sind. Es ist bezeichnend, dass gerade die einstige Zentrale der bürgerlichen Sozialreform, der traditionsreiche 'Verein für Socialpolitik', 1922 zu dem Ort wird, an dem in Weimar zum ersten Mal außerhalb des Unternehmerlagers die 'Krisis der Sozialpolitik' thematisiert wird (Jansen 1998, S. 213-226). Heinrich Herkner, ehemaliger Kathedersozialist und nun zum Wohlfahrtsstaatskritiker gewendeter Vorsitzender des 'Vereins', formuliert hier zum ersten Mal jenen sozialpolitischen Leitsatz, der dann für die Ordoliberalen verbindlich wurde und später von Eucken und Erhard gebetsmühlenhaft wiederholt werden sollte, nämlich dass „eine erfolgreiche Wirtschafts- bzw. Produktionspolitik die beste Sozialpolitik" sei (zitiert nach Janssen 1998, S. 215).

Wenn damit der Sozialstaat aus der Sicht des Bürgertums nicht mehr das Vehikel zur Beförderung gesellschaftlicher Integration war, so ist damit doch - wie wir sehen werden - der Anspruch der 
bürgerlich-liberalen Schichten auf Sozialreform nicht aufgegeben. Dieser Anspruch verlagert sich nun allerdings auf wirt- schaftspolitische 'Ordnungspolitik' plus flankierende Sozialgestaltung und Volkspädagogik. Mit dieser Fortsetzung der Sozialreform im Ordoliberalismus setzt sich auch der „Schulterschluß von Theologie und Nationalökonomie" fort (vom Bruch 1985, S. 101), der für die frühe protestantische Sozialreformbewegung konstatiert worden ist. Zugleich macht die nur auf den ersten Blick paradox anmutende These von der 'Wiedergeburt bürgerlich-protestantischer Sozialreform aus dem Geist der Wohlfahrtsstaatskritik' bei den Ordoliberalen plausibel, warum das ordoliberale Projekt in der Traditionslinie des von den Sozialreformern propagierten Dritten Weges zwischen Kommunismus und Kapitalismus steht, einschließlich der an Schärfe nicht zu überbietenden Ablehnung des 'klassischen' Liberalismus, $(\leftarrow$ S. 184) und warum der Ordoliberalismus die staatspaternalistische Einstellung der bürgerlichen Sozialreform tradiert und damit - so gar nicht liberal - andauernd auf die „,moralische Autorität" des Staates setzt (BonhoefferDenkschrift 1979, S. 94).

In diesem Erklärungsrahmen wird zudem deutlich, dass die frühen Formulie- rungen der ordoliberalen Theorie als Teil einer bürgerlichen Suchbewegung nach stabilitäts- und einheitsstiftenden Kräften verstanden werden müssen, die aus der 'Gruppenanarchie' Weimars (Eucken), dem Chaos der Inflationszeit und der politischen Volatilität hinausführen sollen. Das Bürgertum machte sich auf die Suche nach institutionellen Bollwerken gegen die Bedrohungen der bürgerlichen Ord- nung (Maier 1975), es suchte Schutz vor der „Dynamik der Inflation und dem raschen Wechsel des politischen Geschehens" (Stolleis 1999, S. 111; Mommsen 1987). Die Etablierung der Unabhängigkeit der Reichsbank, die Rückkehr zum Goldstandard, die Sicherung weitreichender Nachprüfungsrechte einer unabhängigen Verfassungsgerichtsbarkeit, überhaupt das zunehmende Ausspielen von Recht (juristisch interpretiert) gegen Gesetz (politisch-positiv gesetzt), abzulesen an der plötzlichen Entdeckung und steilen juristischen Karriere der „Grundrechte" (Stolleis 1999, S. 100-124), die Wiedereinführung der Kapitaldeckung in der Rentenversicherung, schließlich das Hohe Lied auf die 'überindividuellen Schöpfungsordnungen' bei protestantischen Vordenkern wie Paul Althaus oder Emmanuel Hirsch oder das Auflammen der Wertphilosophie - all diese Entwicklungen spiegeln die zeitgenössische Tendenz innerhalb der protestantisch-bürgerlichen Lagers wider, nach institutionellen Bürgen für Stabilität in einer als Bedrohung empfundenen Modeme zu suchen. 


\section{Die protestantische Tiefengrammatik des Ordoliberalismus}

Es ist wichtig festzuhalten, dass die Ordoliberalen sich nicht nur aufgrund enttäuschter wirtschaftspolitischer Erwartungen vom Nazi-Regime abwandten, sondern dass sich ihre schließliche Opposition zunächst aus anderen Quellen speiste: Der unmittelbare Anlass zur Gründung des so genannten 'Freiburger Konzils'2 war der 9. November 1938, die so genannte Reichskristallnacht. Aus tiefer christlicher Sorge wegen des ersten „Wetterleuchtens eines europäischen Krieges (und) der neuen Judenverfolgung" (Ritter 1979, S. 26) konstituiert sich der engere Kreis aus Freiburger Hochschullehrern zunächst nicht, um wirtschaftspolitische Fragen zu diskutieren, sondern um zu einer christlich inspirierten Positionsbestimmung gegenüber dem Nazi-Regime zu kommen. Im Mittelpunkt des Gesprächskreises $(\leftarrow \mathbf{S}$. 185) stehen Fragen des christlichen Widerstandsrechts, des Gehorsamsgebots gegenüber allen weltlichen Obrigkeiten nach Römerbrief 13,1, des Verhältnisses zwischen (lutherischer) Kirche und Staat. Es ging um die ,notwendige Besinnung auf die Aufgaben des Christen und der Kirche in unserer Zeit" (so der Titel der ersten Denkschrift des Freiburger Konzils, siehe Ritter 1979, S. 26-27).

1942 wurde dann auf Bitte von Dietrich Bonhoeffer mit der Ausarbeitung einer Denkschrift für eine mögliche Nachkriegsordnung begonnen. Die Denk- schrift war für eine nach dem Krieg in England einzuberufene Weltkirchenkon- ferenz bestimmt (Blumenberg-Lampe 1973; Ritter 1979). Sie enthielt einen um- fänglichen wirtschaftspolitischen Teil (erarbeitet von Lampe, von Dietze und Eucken), der sich zum Ziel machte, ,Richtschnuren und Verbote" zu formulieren, „, die sich nach unserem Glauben aus Gottes Wort für die Wirtschaft und ihre Ordnung ergeben" (Bonhoeffer-Denkschrift 1979, S. 128).

Viele Überlegungen sind hier zum ersten Mal niedergelegt, die sich später in den Gutachten der Arbeitsgemeinschaft Erwin von Beckerath wiederfinden und die dann schließlich auch die Nachkriegskonzeptionen der Ordoliberalen nach 1945 prägen. Die zunächst rein religiöse Motivation der Freiburger und die durch- gängigen theologischen Reflexionen bei Böhm, Röpke, Rüstow, von Dietze und auch Eucken sind zwar bekannt, in ihren Implikationen für den ordoliberalen Entwurf jedoch m.E. bislang nicht hinreichend beachtet worden. ${ }^{3}$ Dabei ist - so meine These - der ordoliberale

\footnotetext{
${ }^{2}$ Mit den Nationalökonomen von Dietze, Eucken, Lampe [allesamt Mitglieder der Bekennenden Kirche und der Freiburger Christuskirchengemeinde], zusammen mit dem Histori- ker Gerhard Ritter und den Juristen Franz Böhm und Erik Wolf [ebenfalls alle Mitglieder der Bekennenden Kirche] sowie den Pfarrern nebst Frauen der Bekennenden Kirche Dürr, Hesselbacher, Hof, Horch und Frau Weber; vgl. Blumenberg-Lampe (1973, S. 157).

${ }^{3}$ In Reiter/Schmolz (1993) Brakelman/Jänchen (1994), Goldschmidt (1998) und Müller (1997) wird zwar die Bedeutung des christlichen Wertefundaments für die Ordoliberalen zutreffend betont, jedoch nicht näher danach gefragt, inwieweit der ordoliberale Ordnungs- entwurf von der protestantischen Soziallehre in seinen Grundstrukturen geformt ist. Müller (1997), der nach der Möglichkeit einer Rezeption des Ordoliberalismus aus der Perspektive einer 'evangelischen Wirtschaftsethik' fragt, entgeht die eigentliche Pointe: nämlich dass der
} 
Ordnungsentwurf insgesamt nur als Versuch zur Formulierung einer evangelischen Wirtschaftsethik angemessen zu verstehen.

Nun ist die Dominanz der protestantischen Konfessionszugehörigkeit für die deutsche Bildungselite der Zeit typisch (Ringer 1992). Doch wie die skizzierte Frühgeschichte der 'Freiburger Schule' zeigte, ging die Bedeutung des Protestan- tismus für die Ordoliberalen weit über ihre nominale Konfessionszugehörigkeit hinaus. Die Mehrzahl der Ordoliberalen waren religiös tief geprägte protestantische Christen mit enger biographischer Verbindung zur Kirche. Die Bedeutung ihres Glaubens ist deutlich an ihren Veröffentlichungen abzulesen, die immer wieder um das Verhältnis von Religion und Wirtschaft kreisen. Röpke betont in vielfachen Selbstzeugnissen die Bedeutung seiner religiösen Motivation (vgl. Röpke 1976). In den 20er Jahren war Alexander Riistow Mitglied von Paul Tillichs KAIROS- Gruppe, die über eine Verbindung von Christentum und Sozialismus debattierte. Dort traf er mit Eduard Heimann und Adolph Löwe zusammen - Ökonomen, $(\leftarrow \mathbf{S}$. 186) die beide dem religiösen Sozialismus verpflichtet waren. In Rüstows Schriften findet sich bereits früh ein reges Interesse am Thema 'Religion und Wirtschaft'. ${ }^{4}$

Walter Eucken setzt sich in den zwanziger Jahren in einer Reihe von Artikeln in der 'Tatwelt: der Zeitschrift des (Rudolf) Eucken-Bundes, ausführlich mit dem Verhältnis von Sozialismus und Religion und mit der 'geistigen Krise des Kapitalismus' auseinander (Eucken 1926). Dass er überwiegend unter Pseudonym ver- öffentlichte, gab ihm Gelegenheit, sich als „Schüler Rudolf Euckens" zu bekennen und in dessen Namen nach einer ,geistigen Reformation der gesamten Menschheit" zu rufen (Eucken 1926, S. 16). Walter Eucken zeigt sich dem neo-idealistischen Programm seines Vaters verpflichtet, bei dem Positivismus und Idealismus miteinander versöhnt werden sollten, eine Versöhnung, die insbesondere dann gelingen könne, wenn die christliche Religion zum „Gravitationszentrum der Kultur” werde (Graf 1997, S. 58). Bereits 1926 skizziert er erstaunlich prägnant das doppelte, sowohl praktische wie 'transzendentale' Grundmotiv seines später ausgearbeiteten ordnungspolitischen Programms, dem es darum ging, die „Menschen wieder Glieder einer umfassenden geistigen Lebensordnung werden zu lassen und einer solchen Lebensordnung entsprechend die Wirtschafts.form zu gestalten, die dabei aber auch ihrem äußeren Zweck voll entsprechen muß" (Eucken 1926, S. 16; meine Her- vorhebung).

Rüstow interpretiert - ebenso wie Röpke und Eucken - das Scheitern des klassischen Liberalismus auch und vor allem als „religionsgeschichtliches Problem" (Rüstow 1945). Rüstow gilt das laissezfaire Prinzip des klassischen Liberalismus als stoische, deistische „Harmonietheologie" (Rüstow

Ordoliberalismus bereits genau eine solche evangelische Wirtschaftsethik formuliert. Grün- de hierfür liegen vermutlich darin, dass Müller weder die Schriften von Böhm oder von von Dietze näher rezipiert, nocherstaunlicherweise - die Bonhoeffer-Denkschrift.

${ }^{4}$ Siehe sein Schriftenverzeichnis in Rüstow (1963). 
1960, S. 156), die bei Fehlen der entsprechenden sozialen und konfessionellen Voraussetzungen (Calvinismus) zum Scheitern verurteilt war (Rüstow 1945). Im Gegensatz hierzu erklärt Eucken den Untergang des liberalen Prinzips gerade mit dem Mangel an religiöser Wertfundierung - eine Diagnose, die ein bezeichnendes Licht auf das 'metaphysische Fundament' seines eigenen ordnungspolitischen Projekts wirft: „Nicht dadurch verfiel m.E. der Liberalismus, daß er religiösmetaphysisch fundiert war. Im Ge- genteil. Sobald er seinen religiös-metaphysischen Gehalt verlor, verfiel er" (Eucken in einem Brief an Rüstow, zitiert nach Lenel 1991, S. 13).

Müller-Armack verfasst in den frühen 1940er Jahren eine umfassende Studie über Religion und Wirtschaftsstile und wirbt in den 1 950er Jahren für eine neue gesellschaftsintegrierende Irenik zwischen Liberalismus und Marxismus unter dem Dach der christlichen Religion (Müller-Armack 1950). Seine Publikationen aus den vierziger und fünfziger Jahren wie „Das Jahrhundert ohne Gott”, „Über die Macht des Glaubens in der Geschichte" und „Diagnose unserer Gegenwart" zeugen von seinem kontinuierlichen Interesse an religions-soziologischen Fragen und seiner nachhaltigen „protestantischen Prägung" (Müller 1997, S. 29), die er schließlich in der Nachkriegszeit in der Arbeitsgruppe 'Wirtschaft und Soziales' des evangelischen Kirchentages und in vielen anderen kirchlichen Aktivitäten praktisch werden $(\leftarrow$ S. 187) lässt. Constantin von Dietze, später Präses der Gesamtdeutschen Synode der EKD, veröffentlicht 1947 seine Schrift „Theologie und Nationalökonomie", und bei Böhm ist die religiöse Motivation ebenfalls unübersehbar (Nörr 1993). Die Freiburger Bonhoeffer-Denkschrift, mitverfasst von Böhm, Eucken, und von Diet- ze, ist schließlich der explizite Versuch der Formulierung einer protestantischen Soziallehre, einer christlich fundierten Lehre der Wirtschafts- und Gesellschaftsordnung.

\section{4. ,,[...\}. die freie, natürliche, gottgewollte Ordnung verwirklichen" ${ }^{5}$}

Wenn es zutrifft, dass in der Bonhoeffer-Denkschrift des Freiburger Kreises die ,geistigen Wurzeln der Sozialen Marktwirtschaft" vorformuliert sind (von Bismarck 1979, S. 153) bzw. dass die Denkschrift die „Essenz des ordoliberalen Denkens" enthält (Horn zitiert nach Goldschmidt 1998, S. 35), dann muss nach der Stärke des Einflusses dieser 'geistigen Wurzeln' auf den Ordoliberalismus gefragt werden. Programmatisch hatte Franz Böhm bereits in seinem Beitrag zur 'Ordnung der Wirtschaft'-Schriftenreihe festgehalten: Eine Wirtschaftsordnung ist nicht nur eine „technische, sondern auch eine sittliche Ordnung" (Böhm 1937, S. 7). Wie sollte diese Ordnung ausgestaltet sein?

\footnotetext{
${ }^{5}$ Eucken (1952, S. 176).
} 
Was ist die Quelle ihrer Sittlichkeit?

Einige von Böhms Gedanken hierzu finden sich formuliert in einer späteren, bereits in die Nachkriegszeit fallende Rede vor dem evangelischen Arbeitskreis der CDU (Böhm 1960; Nörr 1993). Bezeichnenderweise entwickelt Böhm hier seine Gedanken anhand des Gleichnisses vom barmherzigen Samariter. Doch im Mittelpunkt seiner Interpretation steht nicht, wie man erwarten könnte, die praktische Nächstenliebe; vielmehr dient ihm das Gleichnis dazu, auf den christlichen Humanismus hinzuweisen, der in einer funktionierenden Verkehrswirtschaft bereits verkörpert sei. Am Beispiel der Fürsorge des Samariters für den Überfallenen diskutiert Böhm die Frage, ob bzw. inwiefern eine geschützte Eigentums- und Rechtsordnung christlichen Ziele dienlich oder ihnen abträglich ist.

Die zentrale Frage für Böhm ist, inwieweit eine Wirtschaftsordnung es dem Gerechten erlaubt, gerecht zu sein, und dem es Sünder erschwert, in Sünde zu leben. Für Böhm sind zunächst alle Ordnungen, auch totalitäre, ethisch gleichwertig, da sie als mögliche „Bauformen der Gesellschaft [...] sozusagen kraft Schöpfungsanlage zu unserer Disposition stehen" (ebd., S. 12). Erst in einem zweiten Schritt geht es dann darum zu fragen, in welchem Maße unterschiedliche Ordnungen der besonderen Sündenverfallenheit der Menschen Rechnung tragen. Das impliziert eine klare Zuschreibung von Schuld zwischen Individuum und Institution (Nörr 1993, S. 12). Die Ordnungen 'an sich' sind unschuldig, sie sind bloß mehr oder weniger gut geeignet, die allgegenwärtigen „Versuchungen zu Lieblosigkeit und Bosheit" (Böhm 1960, S. 18) unter Kontrolle zu halten. Die $(\leftarrow$ S. 188) Ordnungen sind in einem stärkeren oder schwächeren Ausmaß verwundbar durch böse Absichten der Menschen, durch den Missbrauch der Ordnung: „Man kann ein Beil dazu benutzen, Holz zu spalten oder einen Menschen zu töten. An dem Baugedanken des Beils ändert sich dadurch nichts" (ebd., S. 12).

Wenn Böhm in diesem Zusammenhang explizit die totalitäre Herrschaft als bedenkenswerte Gesellschaftsordnung ansieht, die moralisch den anderen 'Bauformen' zunächst weder unter- noch überlegen sei,6 so ist dies (formuliert im Deutschland der 1950er Jahre von einem Wissenschaftler, Politiker, Juristen und strenggläubigen Protestanten, der als Beauftragter der Adenauer-Regierung die 'Wiedergutmachungs'-Verhandlungen mit Israel führt) ein beeindruckender Beleg dafür, welche langen Umwege beschritten werden mussten, ehe der protestantische Ordoliberale sich schließlich argumentativ zur freiheitlichen Privatrechtsordnung durchringt. Den klassisch liberalen Weg über die Postulierung unveräußerlicher und 'sakrosankter' individuell-subjektiver Freiheitsrechte beschreitet

\footnotetext{
${ }^{6}$,Wahrscheinlich werden wir zu dem Ergebnis kommen, daß die totalitäre Diktatur nicht an sich böse und vermessen ist, daß sie aber eine Versuchung zum Bösen und zur Vermessenheit in einem Stärkegrad enthält, der auch gläubige Christen nicht gewachsen sind" (Böhm 1960, s. 13).
} 
Böhm ganz bewusst nicht, sind doch diese Freiheitsrechte im Ordoliberalismus mit dem ve- hement abgelehnten Individualismus, dem 'Paläo-Liberalismus', mit Manchester- tum und damit mit sozialer und wirtschaftlicher Anarchie gleichgesetzt (Nörr 1993).

Hier kommt eine Arglosigkeit gegenüber staatlichen Zwangsstrukturen zum Vorschein, die für die Ordoliberalen insgesamt typisch ist. Sie ist allerdings nicht pauschal aus einer Staatsfixierung des Luthertums zu erklären. In der Bonhoeffer- Denkschrift hatten die Freiburger explizit gegen die neulutherische Deutung der Obrigkeit Stellung bezogen, wie sie Anfang der 1930er Jahre zugespitzt etwa von Friedrich Gogarten oder Paul Althaus formuliert wurde: „Obrigkeit, wo sie ist, [ist] von Gott verordnet" (Gogarten 1932, S. 109) oder: „Das Grundwort der Politik ist also: Gehorsam" (Althaus 1931, S. 45). Die Denkschrift betont vielmehr als Ziel, die ,lutherische Lehre von der christlichen Obrigkeit [...] für unsere Zeit fortzubilden" (Bonhoeffer-Denkschrift 1979, S. 67). Doch selbst einem der damals Beteiligten wird in der Rückschau klar, wie wenig liberal, sondern ausgesprochen staatsfixiert das Ergebnis ausfiel: „,der so gezeichnete Staat war zwar alles andere als totalitär, aber doch mit Aufgaben überfrachtet und gleichsam in eine Superstaatlichkeit hinein ausgeweitet" (Thielicke 1979, S. 18; Hervorhebung im Ori- ginal). Fragen wir also: Warum fiel auch der ordoliberale Versuch einer ethischen Neubestimmung von Staats- und Wirtschaftsordnung, explizit gerichtet gegen das totalitäre Nazi-Regime, erneut so obrigkeitsfixiert aus?

Was die Freiburger Schrift von Althaus' schlichter Heiligsprechung einer jeden weltlichen Ordnung unterschied, war nur, dass man an Stelle des unbedingten Gehorsamsgebotsdie Christenpflicht setzte, die lutherische Obrigkeitslehre 'für unsere $(\leftarrow$ S. 189) Zeit fortzubilden' (s.o.). Zwar stand auch bei Constantin von Dietze als erster protestantischer Reflex erwartungsgemäß die pure Anerkennung jeder Ordnung als Schöpfungsordnung. Der Christ sei verpflichtet, diese Ordnungen zu ehren, zu respektieren - doch eben auch, so heißt es schüchtern, sie weiterzuentwickeln:

„Eine Welt, auch eine der Sünde verfallene Welt, die der Schöpfer noch erhält, muß aber in sich gewissen Ordnungen folgen, und es ist die Pflicht der Christen, diesen Ordnungen nachzuspüren, ihnen die tatsächlichen Maßnahmen anzupassen, die zur Regelung der Wirtschaft ergriffen werden" (von Dietze 1947, S. 31-32; meine Hervorhebung). In den Worten Euckens: Wirtschaftspolitik muss so gestaltet sein, dass sie die „freie, natürliche, gottgewollte Ordnung verwirklicht" (Eucken 1952, S. 176). Dabei herrscht bei von Dietze (wie bei Eucken und Böhm) die Vorstellung vor, dass sich die natürliche gottgewollte Ordnung 'erschauen' lässt, dass die den wirtschaftlichen Gesetzmäßigkeiten zugrundeliegende „,verborgene Kraft" (Böhm 1960, S. XLVIII) sich der schauenden Vernunft 'zeigt'. Böhm schreibt in diesem Zusammenhang, dass ,in einer Gesellschaft von Individuen, die in individueller Freiheit gesellschaftlich zusammenleben, von der Gesellschaft her betrachtet, die 
schöpfungsmäßigen Ordnungstafeln und Wegweiser aufleuchten" (Böhm 1954, S. 99). ${ }^{7}$

Geleitet werden kann die Anpassung an die erschaute Ordnung aus Sicht der Ordoliberalen nur durch das ,verpflichtende Bekenntnis zu ewigen, letzten Wert- prinzipien, die für das Abendland in der christlichen Offenbarung beschlossen liegen" (Müller-Armack 1981, S. 316). Das klingt wenig spezifisch, doch enthält die Bonhoeffer-Denkschrift eine recht klare Darlegung der praktischen Konse- quenzen dieses 'verpflichtenden Bekenntnisses'. Jenseits der „Sachnotwendigkeiten" (Bonhoeffer-Denkschrift 1979, S. 128), zu denen die christliche Glaubensüberzeugung nicht Stellung zu beziehen habe, hat die stark religiöse Färbung des ordoliberalen Ordnungsentwurfs ihren Ausgangspunkt im skeptischen Menschenbild des lutherischen Protestantismus. Diesem Menschenbild zufolge ist der Mensch immer zugleich Gerechter und Sünder. Die Ordnungen dienen dazu, die sündhafte Seite unter Kontrolle $\mathrm{zu}$ bringen und der gerechten Seite Entfaltungsmöglichkeiten zu bieten. Hier wird die - modern gesprochen - Anreizmechanik einer Wirtschaftsordnung durch eine konfessionelle Soziallehre fundiert. Constantin von Dietze betont in diesem Zusammenhang, dass die nationalökonomische Theorie sich nicht, ,alle Menschen als Engel oder Teufel vorstellen" darf (1947, S. 26). Mit anderen Worten, die wirtschaftliche Ordnung muss in ihrer konkreten Ausgestaltung berücksichtigen, dass die Menschen ,allesamt Sünder vor Gottes Gericht, aber doch nicht gewissenlos [sind]" (Bonhoeffer-Denkschrift 1979, S. 72).

Das für das Verständnis des Ordoliberalismus zentrale skeptische Menschenbild des lutherischen Protestantismus tritt klarer hervor, wenn man es kontrastiert mit $(\leftarrow \mathbf{S}$. 190) dem viel optimistischeren 'gnadengewissen' Bild der nordamerikanischen neu-calvinistischen und freikirchlichen Strömungen und Sekten - einschließlich des hieraus abgeleiteten liberalen Staats- und Wirtschaftsordnungskonzepts (Troeltsch 1994 [1912], S. 757). Hier findet sich im Unterschied zum deutschen Ordoliberalismus nun tatsächlich eine individualistische Fundierung politischer und wirtschaftlicher Freiheiten. Die im Neu-Calvinismus und in den protestantischen Sekten vorherrschenden Vorstellungen von der „Unverlierbarkeit des Gnadenstandes” (ebd., S. 622) führen zu einer „,individualistischen und rein utilitaristischen Auffassung des Staates", der hieraus abgeleitete „,politische Individualismus [steht] nun dem lutherischen Staatskirchentum geradezu diametral gegenüber" (ebd., S. 757; vgl. Gogarten 1932, S. 122). Diese Unterschiede in Menschenbild und Staatsverständnis prägen entsprechend deutlich die Ordnungsentwürfe der klassischen Liberalen einerseits und der Ordoliberalen andererseits.

Ist nach der Lehre der neu-englischen Sekten die Gemeinschaft der Christen eine Gemeinschaft der Gerechten, so ist das Ausmaß legitimer Intervention von außen in ihre Geschäfte von vornherein als

\footnotetext{
${ }^{7}$ Wie sehr dieser Glaube an eine objektiv 'erkennbare', vorgegebene Wertordnung vom Neukantianismus und der Wertphilosophie beeinflusst war, ist m.E. plausibel von Müller (1997, S. 36-41) herausgearbeitet worden.
} 
äußerst begrenzt konzipiert. Die Notwendigkeit der Kontrolle gilt hier primär dem Missbrauch staatlicher Macht. Ansonsten genießt die Gemeinschaft volle Freiheit zur Regelung ihres Binnenverhältnisses. Ist hingegen der Mensch, selbst der gläubige und fromme Christ, unabänderlich und immerzu auch sündig, so liegt die Betonung der Kontrolle auf dem freien Handeln des Menschen, das, sich selbst überlassen, in Verderbnis führen kann. Aus dieser Sicht ist es der Markt als freies Zusammenkommen der Menschen, nicht der Staat, der ethisch reguliert, diszipliniert und kontrolliert werden muss. Genau in diesem Sinne gründet der ordoliberale Entwurf auf der Überzeugung, dass die „Menschen nicht gut genug [sind], um die Vereinigung aller politischen und wirtschaftlichen Macht recht zu gebrauchen oder um sich in völlig freier Wirtschaft der Ausbeutung zu enthalten" (von Dietze, zitiert nach Brakelmann/Jän- nichen 1994, S. 366). Sie sind aber auch nicht „,so schlecht, daß man sie staatlicher Tyrannei oder privater Macht unterwerfen dürfte oder gar müßte" (ebd.). Als Mittelweg bietet sich die staatliche Disziplinierung der Wirtschaftssubjekte an. Wenn man danach fragt, was die staatliche Ordnung als Menschenwerk - und damit selbst ein Werk von Sündern - in die Lage setzen soll, das sündhafte Handeln der Menschen zu kontrollieren, so finden sich in der ordoliberalen Konzeption zwei Antworten. Zum einen wird Hoffnung in Mechanismen der Koordination und Kontrolle gesetzt, wie in den Preismechanismus oder in Rechtsverfahren, die sich gegenüber dem diskretionären Handeln 'mit böser Absicht' als immun erweisen sollen. Die 'Spielregeln' des Wettbewerbs müssen als „,Veranstaltungen der Rechtsordnung" (Böhm 1937, S. 120) dem manipulativen, i.e. politischen Zugriff entzogen werden. Doch was hier vordergründig als liberal erscheint, ist nicht Skepsis gegenüber dem Staat und den staatlichen Ingerenzen in die freie Vergemeinschaf- tung seiner Bürger, sondern Skepsis gegenüber den Menschen, zum rechten Gebrauch von (staatlicher) Macht überhaupt fähig zu sein, gepaart mit der Überzeugung, $(\leftarrow$ S. 191) dass die staatliche Macht dennoch unverzichtbar sei gerade wegen der menschlichen Willensschwäche. Die Konsequenz ist daher im Ordoliberalismus das Plädoyer für einen mit weitreichenden Interventionsrechten ausgestatteten Staat, der selbst unabhängig ist vom je aktuellen politischen oder gesellschaftlichen Wollen. Die Bedeutung non-majoritärer Institutionen in der deutschen politischen Ökonomie leitet sich aus dieser Weltsicht ab. Verbunden ist diese Vorstellung zum anderen mit einem strikt elitären Demokratiekonzept. Damit der Staat eine „ethische und erzieherische Potenz" sein kann (Bonhoeffer-Denkschrift 1979, S. 103), muss als „Idealziel schöpferischer Politik der Zukunft [...] aus der unterschiedslosen Masse eine neue Notabelnschicht der politisch Einsichtigen und sittlich Zuverlässigen" herausgebildet werden (ebd., S. 74). Erst dadurch kann der Staat als unabhängige und sittlich superiore Instanz gedacht werden. Und an dieser Stelle sind die Ordoliberalen strikt neu-lutherisch: „Der Staat [...] muß auf Autorität gegründet sein, nicht auf Majorität" (Althaus 1936, S. 29). 
Wenn man diese skeptische Anthropologie des Luthertums in Rechnung stellt, ist es nicht mehr überraschend, daß die Ordoliberalen - vollkommen unliberal - dem Staat andauernd die Aufgabe einer „personenbildenden Volkserziehung” (Bonhoeffer-Denkschrift 1979, S. 74) zuschreiben. „Der Mensch ist aus der Gnade gefallen und von selbstsüchtigen Trieben beherrscht; er bedarf darum des Zwangs und der Strafe. Aber er hat das sittliche Bewußtsein keineswegs völlig verloren; es bedarf freilich der Ausrichtung, Klärung, Festigung, Erziehung" (ebd., S. 72). Hier liegt der volkspädagogische Anspruch des Ordoliberalismus und die enorme ethische Aufladung des Staates als Moralanstalt begründet.

Die Konsequenz der Vorstellung vom 'radikalen Verfallensein des Menschen an die Macht des Bösen' (Gogarten) ist die Forderung, eine Wirtschaftsordnung (staatlich!) so einzurichten, dass sie „den denkbar stärksten Widerstand gegen die Macht der Sünde ermöglicht" und dass „es den Wirtschaftenden nicht unmöglich gemacht oder systematisch erschwert wird, ein Leben evangelischer Christen zu führen" (Bonhoeffer-Denkschrift 1979, S. 129). Die Wettbewerbsordnung wird von den Ordoliberalen als Disziplinierungsinstrument verstanden, das die Sünde etwa den „Mammondienst", den „Dämon der Habgier" (ebd., S. 135) - in die Schranken verweisen soll. Der Markt, beaufsichtigt vom Staat, ist das disziplinie- rende Koordinationsmittel, das die Berufs- und Arbeitsaskese hervorbringt, die bei den neu-englischen Sekten immer schon individuell vorausgesetzt ist. Für die Ordoliberalen ist der Preismechanismus in erster Linie nicht ein Mittel zur effizienten Faktorallokation, sondern ein Disziplinierungsinstrument für die willensschwachen Wirtschaftsakteure in dem,,wirtschaftliche[n] Alltag mit seinen alltäg- lichen Versuchungen" (Böhm 1937, S. 12).

Dabei geht es auch um die Vorstellung, dass sowohl das Persönlichkeitsrecht eines jeden Einzelnen (hergeleitet aus dem Argument des imago Dei) als auch gleichzeitig die Sündhaftigkeit eines jeden Einzelnen die Forderung nach einer Gesellschaftsordnung begründen, die frei von der Herrschaft des Menschen über $(\leftarrow$ S. 192) den Menschen, aber dabei doch nicht herrschaftsfrei ist. Das ORDOKonzept entwirft folglich eine Sozialordnung, „die zwar den Menschen in ihre eigene Gesetzlichkeiten einspannt, ihn zum Ausgleich dafür aber von der Unterwerfung unter die Botmäßigkeit eines von Menschen gehandhabten Regimes freistellt" (Böhm 1960a, S. XLVIIII). Die Wirtschaftsund Sozialordnung soll nach dieser Vorstellung vor allem den „menschlichen Trieb zur Beherrschung, Unterdrückung und Ausbeutung von seinesgleichen" begrenzen (Böhm 1960a, S. LXIII). Die Legitimierung der Marktwirtschaft erfolgt hier also gerade nicht über das klassisch liberale Argument (das größte Glück der größten Zahl). Vielmehr geht es darum, die Möglichkeit zur Herrschaft des Menschen über den Menschen zu minimieren (der Wettbewerb als 'geniales Entmachtungsinstrument', Böhm). Rüstow stellt explizit fest, dass sich „um der Realisierung 
ethischer Werte willen auch starke Einbußen an wirtschaftlicher Effizienz rechtfertigen lassen" (zitiert nach Müller 1997,

S. 14). Hier ist die „Grenze zwischen Markttheorie und Sozialethik[...] verwischt” (Nawroth 1961, S. 129), und sie soll verwischt sein. Denn die von den Ordoliberalen angestrebte Wirtschaftsordnung wird explizit gedacht als Verwirklichung eines „,sozialen und ethischen Ordnungswollens" (Eucken 1952, S. 370). Erneut begegnet uns hier das Programm Rudolf Euckens der Versöhnung von Positivismus und Idealismus in Gestalt einer wirtschaftspolitischen Verbindung „ökonomischer Sachlogik" mit einem ,sozial-ethischen Wollen" (Eucken 1952, S. 370) auf christlichem Fundament. Hierzu gehört auch, dass der von den Ordoliberalen propagierte 'Dritte Weg' zwischen Kollektivismus und Anarchie sich auf das theologisch hochgradig aufgeladene Konzept der Person gründet, das in klarer Entgegensetzung sowohl zum atomisierten, selbstgerechten Individuum, als auch zum Kollektiv steht. Es ist bezeichnend, dass die Ordoliberalen es konsequent vermeiden, von individuellen Rechten zu sprechen, sondern sich mit dem Konzept der Person selbst zwischen dem marxistischen Kollektivismus und dem anarchischen Individualprinzip des klassischen Liberalismus verorten. Man wähnt sich in Äquidistanz zu beiden großen Heilslehren der Gegenwart und glaubt, mit dem Konzept der christlichen Person den Boden für eine 'neue Irenik', für einen zweiten Westfälischen Frieden zwischen den polaren Weltanschauungen geschaffen zu haben (MüllerArmack 1950; vgl. Rüstow 1960, insbes. S. 176-178). Dieser Mittelweg, der die Extreme des Liberalismus und des Kollektivismus zu vermeiden verspricht, - so meine These - ist im Wesentlichen religiös begründet, auch wenn Rüstow behauptet: „Wir Neoliberalen haben [...] gar keine eigene Theologie" (Rüstow 1960, S. 176). ${ }^{8}(\leftarrow$ S. 193)

\section{Die protestantische Ethik und der Geist der sozialen Marktwirtschaft}

Ich sehe drei Konsequenzen meiner Argumentation, die ich abschließend kurz skizzieren möchte. ${ }^{9}$ Eine erste Schlussfolgerung ist, dass all die staatlichen Marktbeschränkungen, die 'ethische Regulierung' der Wirtschaft durch den Staat, die institutionalisierte Skepsis gegenüber der Freiheit des Individuums, dazu der Anspruch auf umfassende Gesellschaftspolitik und die volkspädagogischen Anwandlungen - dass all dies integraler Bestandteil der ordoliberalen Wirtschafts- und Gesellschaftskonzeption bis in die 1960er Jahre hinein bleibt. Ordoliberalismus ist

\footnotetext{
${ }^{8}$ Walter Benjamin schreibt: ,,der Kapitalismus [ist] eine reine Kultreligion [...] Es hat in ihm alles nur unmittelbar mit Beziehung auf den Kultus Bedeutung, er kennt keine spezielle Dogmatik, keine Theologie" (Benjamin [1921] 1985, S. 100), Darüber wäre erneut zu diskutieren.

${ }^{9}$ Für eine ausführlichere Diskussions. Manow (2000; 2001, Kapitel 3).
} 
eine Lehre von der 'Korrektur der Wirtschaft durch die Sittlichkeit', die zunächst wenig liberal durchwirkt ist. Es war eine Selbsttäuschung anzunehmen, Deutschland sei mit dem Durchbruch des Ordoliberalismus als Wirtschaftsdoktrin bereits 'im Westen angekommen'. Ein Dritter Weg zwischen Kommunismus und Kapitalismus in der Tradition der bürgerlichen Sozialreform bleibt bis weit in die Nachkriegszeit die leitende Vorstellung der Ordoliberalen, und dieses Votum für einen Mittelweg zwischen Ost und West wäscht sich erst allmählich im westintegrierten Wirtschaftswunderdeutschland aus.

Zweitens nimmt meine Interpretation der für die 1950er Jahre oft vermerkten „Diskrepanz zwischen liberaler Doktrin und wirtschaftspolitischer Realität des Staatsinterventionismus und der Planung" etwas ihre Rätselhaftigkeit (Abelshauser 1976, S. 415, Fn. 3, m.w.L.). Zumindest lässt sich diese Diskrepanz nicht mehr als simpler Widerspruch zwischen hehren Prinzipien und alltäglicher politischer Praxis abtun. Das vielfältige Abweichen von der Linie einer liberalen Wirtschaftspolitik so meine These - lässt sich erst angemessen verstehen, wenn man gewahr wird, dass diese 'liberale Linie' äußerst verschwommen war. Ludwig Erhards Votum für die Ausgabe von rabattmarkenähnlichen „Aufbaumarken" zur Finanzierung der Investitionshilfe für die Ruhr (Adamsen 1981, S. 114-115) mit dem Ziel einer breiten Vermögensstreuung und Bestrafung des Luxuskonsums ist nur eines von vielen Beispielen für die praktischen Konsequenzen einer Weltanschauung, die zum regulierenden Eingriff ins freie Marktgeschehen in sozialgestalterischer Absicht nicht erst lange überredet werden musste. Auch der Staatspaternalismus in Müller-Armacks Plädoyer für eine 'zweite Phase der sozialen Marktwirtschaft' oder Erhards Konzept einer 'formierten Gesellschaft' sind dann nicht einfach nur ordoliberale 'Betriebsunfälle', sondern stehen deutlich in der Kontinuität eines pro- testantisch eingefärbten Staatsverständnisses, das schon 1937 der Regierung die Aufgabe einer 'gewaltigen nationalen Erziehungsarbeit' (s.o.) zugewiesen hatte. Wo man weder dem einzelnen Wirtschaftssubjekt traute, noch der 'Sakramentsmagie' intermediärer Instanzen, blieb nichts anderes übrig als die Vorstellung, der Staat müsse als moralische Autorität dafür Sorge tragen, dass den Bürgern jene $(\leftarrow$ S. 194) Sittlichkeit vermittelt wird, ohne die Freiheit und Menschenwürde in einer Markt- wirtschaft nicht existieren könnten. Mit den elementaren Prinzipien des politischen und ökonomischen Liberalismus hatte das alles wenig gemein.

Drittens kann meine Argumentation auch neue Akzente in der Interpretation der bundesdeutschen Nachkriegsordnung setzen. Die Ablehnung einer 'Vorfahrtsregelung' für die individualistische Glücksverfolgung, die Polemik gegen das liberale laissez-faire, die Sorge um relative Einkommensgleichheit und der paternalistische Grundzug der Wirtschafts- und Sozialpolitik sowie die volkserzieherischen Momente wurden hier als Kennzeichen einer protestantischen Soziallehre identifiziert, eingekleidet in eine vorgeblich liberale Wirtschaftsdoktrin. Aus dieser Perspektive ist 
dann die Abweichung des kontinental-europäischen Sozial- und Wirtschaftsmodells vom Marktmodell angelsächsischer Prägung nicht mehr nur aus dem Einfluss der katholischen Soziallehre zu erklären. Dies jedoch ist die gängige Interpretation. Üblicherweise wird die politische Ökonomie Nachkriegsdeutschlands als Kompromiss zwischen Wirtschaftsliberalismus einerseits und katholischem Wohlfahrtsstaat andererseits charakterisiert - eine Interpretation, die nicht nur auf den deutschen Fall angewendet wird. In der Literatur wird der „soziale Kapitalismus" Kontinentaleuropas generell 'katholisch' gedeutet (s. Esping-Andersen 1990; Kersbergen 1995). Allein „Christian Democracy" - und das meint vor allem die dominant katholischen Parteien wie die CDU, die italienische DC oder die niederländische Katholieke Volkspartij - ist nach dieser Interpretation neben der sozialistischen Arbeiterbewegung eine für die Abweichung des europäischen Sozialmodells vom rein markt-liberalen Modell angelsächsischer Prägung relevante Kraft gewesen (Huber/Ragin/Stephens 1993). Diese Sichtweise unterschlägt jedoch den genuin lutherischprotestantischen Einfluss eines sozialreformerischen Bürgertums, das in den gängigen Darstellungen pauschal dem Liberalismus-Lager zugeschlagen wird. Ich halte das für irreführend, weil damit der spezifisch anti-liberale Charakter dieses 'Liberalismus' ignoriert wird und damit der konfessionelle Kompromisscharakter der politischen Ökonomien Kontinentaleuropas weitgehend unverstanden bleibt.

\section{Literatur}

Abelshauser, Werner, 1976: Freiheitlicher Sozialismus oder soziale Marktwirtschaft? Die Gutachtertagung über Grundfragen der Wirtschaftsplanung und Wirtschaftslenkung am 21. und 22. Juni 1946, in: Vierteljahreshefte für Zeitgeschichte 24, S. 415-449.

Abelshauser, Werner, 1984: Wirtschaftliche Wechsellagen, Wirtschaftsordnung und Staat: Die deutschen Erfahrungen, in: Dieter Grimm (Hrsg.), Staatsaufgaben, Frankfurt a.M.: Suhr- kamp, S. 199-232.

Adamsen, Heiner R., 1981: Investitionshilfe für die Ruhr. Wiederaufbau, Verbände und Soziale Marktwirtschaft 1948-1952, Wuppertal: Peter Hammer.

Althaus, Paul, 1931: Staatsgedanke und Reich Gottes, Langensalza: Beyer \& Söhne. Althaus, Paul, 1936: Obrigkeit und Führertum, Gütersloh: Bertelsmann. $(\leftarrow$ S. 195)

Benjamin, Walter, [1921] 1985: Kapitalismus als Religion, in: Walter Benjamin, Gesammelte Schriften VI, hrsg. von Rolf Tiedemann und Hermann Schweppenhäuser, Frankfurt a.M.: Suhrkamp, S. 100-103.

Bismarck, Philipp von, 1979: Nachwort, in: In der Stunde Null. Die Denkschrift des Freiburger 
Bonhoeffer-Kreises, Tübingen: Mohr (Siebeck), S. 153-156.

Blumenberg-Lampe, Christine, 1973: Das wirtschaftspolitische Programm der 'Freiburger Kreise'. Entwurf einer freiheitlich-sozialen Nachkriegswirtschaft, Nationalökonomen gegen den Nationalsozialismus, Berlin: Duncker \& Humblot.

Böhm, Franz, 1937: Die Ordnung der Wirtschaft als geschichtliche Aufgabe und rechtsschöpferische Leistung. Heft 1, Ordnung der Wirtschaft, Schriftenreihe herausgegeben von Franz Böhm, Walter Eucken und Hans Grossmann-Doerth, Stuttgart/Berlin: Kohlhammer.

Böhm, Franz, 1960 [1957]: Die verantwortliche Gesellschafr, in: Franz Böhm, Reden und Schriften, hrsg. von Ernst-Joachim Mestmäcker, Karlsruhe: C.F. Müller, S. 3-22.

Böhm, Franz, 1960a: Die Idee des ORDO im Denken Walter Euckens, in: ORDO 3, S. XV-LXIV:

Bonhoeffer-Denkschrift, 1979. Hier zitiert nach: In der Stunde Null. Die Denkschrift des Freiburger Bonhoeffer-Kreises, Tübingen: Mohr (Siebeck).

Brakelmann, Günter und TraugottJännichen (Hrsg.), 1994: Die protestantischen Wurzeln der Sozialen Marktwirtschaft. Ein Quellenband, Gütersloh: Gütersloher Verlagshaus.

Der Weg in die Soziale Marktwirtschaft. Referate, Protokolle, Gutachten der Arbeitsgemein- schaft Erwin von Beckerath 1943-1947. Bearbeitet und herausgegeben von Christine BlumenbergLampe, 1986, Stuttgart: Klett Cotta.

Dietze, Constantin von, 1947: Theologie und Nationalökonomie. (Das christliche Deutsch- land. 1933 bis 1945; Evangelische Reihe Heft 8), Tübingen/Stuttgart: Furche.

Esping-Andersen, Gösta, 1990: Three Worlds ofWelfare Capitalism, New York: Polity Press.

Eucken, Walter, 1926: Die geistige Krise und der Kapitalismus, in: Die Tatwelt, S. 13-16 [unter dem Pseudonym Dr. Kurt Heinrich].

Eucken, Walter, 1932: Staatliche Strukturwandlungen und die Krisis des Kapitalismus, in: Weltwirtschafrliches Archiv 36, S. 297-321.

Eucken, Walter, 1952: Grundsätze der Wirtschaftspolitik, Tübingen: Mohr (Siebeck). Gogarten, Friedrich, 1932: Politische Ethik. Versuch einer Grundlegung, Jena: Eugen Diederichs.

Goldschmidt, Nils, 1998: Christlicher Glaube, Wirtschaftstheorie und Praxisbezug. Walter Eucken und die Anlage 4 der Denkschrift des Bonhoeffer-Kreises, in: Historisch-Politische Mitteilungen. Archiv für Christlich-Demokratische Politik, 5. Jg., Köln: Böhlau, S. 33-48. Graf, Friedrich Wilhelm, 1997: Die Positivität des Geistigen. Rudolf Euckens Programm neoidealistischer Universalintegration, in: Gandolf Hübinger, Rüdiger vom Bruch und Friedrich Wilhelm Graf (Hrsg.), Kultur und Kulturwissenschaften um 1900 II: Idealismus und Positivismus, Stuttgart: Franz Steiner, S. 53-85.

Haselbach, Dieter, 1991: Autoritärer Liberalismus und Soziale Marktwirtschaft. Gesellschafr und 
Politik im Ordoliberalismus, Baden-Baden: Nomos.

Heller, Hermann, 1933 [1992]: Autoritärer Liberalismus, in: Die Neue Rundschau, 44. Jg., H. 1, S. 289-298. Hier zitiert nach Herman Heller, 1992: Gesammelte Schriften. Zweiter Band: Recht, Staat, Macht, Tübingen: Mohr (Siebeck), S. 643-653.

Hentschel, Volker, 1996: Ludwig Erhard: Ein Politikerleben, München: Olzog.

Herbst, Ludolf, 1982: Der Totale Krieg und die Ordnung der Wirtschaft: die Kriegswirtschafr im Spannungsfeld von Politik, Ideologie und Propaganda, 1939-1945, Stuttgart: Deutsche VerlagsAnstalt.

Holmes, Stephen, 1993: The Anatomy of Antiliberalism, Cambridge, Mass.: Harvard University Press. $(\leftarrow$ S. 196)

Huber, Evelyne, Charles Ragin und John D. Stephens, 1993: Social Democracy, Christian Democracy, Constitutional Structure and the Welfare State, in: American Journal of Sociology 99, No. 3, S. 711-749.

Janssen, Hauke, 1998: Nationalökonomie und Nationalsozialismus. Die deutsche Volkswirtschaftslehre in den dreißiger Jahren, Marburg: Metropolis.

Kersbergen, Kees van, 1995: Social Capitalism. A Study of Christian Democracy and the Welfare State, London: Routledge.

Krohn, Claus-Dieter, 1981: Wirtschaftstheorien als politische Interessen. Die akademische Nationalökonomie in Deutschland 1918-1933, Frankfurt a.M.: Campus.

Lenel, Hans Otto, 1991: Walter Euckens Briefe an Alexander Rüstow, in: ORDO 42, S. 11-14.

Maier, Charles, 1975: Recasting Bourgeois Europe. Stabilization in France, Germany, and Italy in the Decade after World War I, Princeton, N.J.: Princeton University Press.

Manow, Philip, 2000: 'Modell Deutschland' as an inter-denominational Compromise. Discussion Paper 1/2000. Minda-de-Gunzburg Center for European Studies, Harvard University, Cambridge, Mass.

Manow, Philip, 2001: Social Protection, Capitalist Production. The German Political Economy and the Bismarckian Welfare State from the 1880s to the 1990s. Habilitationsschrift, Konstanz.

Mommsen, Hans, 1987: Die Auflösung des Bürgertums seit dem späten 19. Jahrhundert, in: Jürgen Kocka (Hrsg.), Bürger und Bürgerlichkeit im 19. Jahrhundert, Göttingen: Vanden- hoeck \& Ruprecht, S. 288-315.

Müller, Eckart, 1997: Evangelische Wirtschaftsethik und Soziale Marktwirtschaft. Die Konzeption der Sozialen Marktwirtschaft und die Möglichkeiten ihrer Rezeption durch eine evangelische Wirtschaftsethik, Neukirchen: Neukirchener Verlag.

Müller-Armack, Alfred, 1950: Soziale Irenik. Über die Möglichkeit einer die Weltanschauungen 
verbindenden Sozialidee, in: Weltwirtschaftliches Archiv 64. Hier zitiert aus dem Nachdruck in Alfred Müller-Armack, Religion und Wirtschaft. Geistesgeschichtliche Hintergründe unserer europäischen Lebensform, Bern/Stuttgart: Paul Haupt, S. 559-578. Müller-Armack, Alfred, 1981: Diagnose unserer Gegenwart: Zur Bestimmung unseres geistesgeschichtlichen Standorts, 2. Aufl., Bern/Stuttgart: Paul Haupt.

Nörr, Knut W., 1993: An der Wiege deutscher Identität nach 1945: Franz Böhm zwischen Ordo und Liberalismus (Schriftenreihe der Juristischen Gesellschaft zu Berlin, Heft 129), Berlin: de Gruyter. Rieter, H. und M. Schmolz, 1993: The Ideas of German Ordoliberalism 1938-1945: Pointing the Way to a new Economic Order, in: European Journal of the History of Economic Thought 1, S. 87-114. Ringer, Fritz, 1992: A Sociography of German Academics, 1863-1938, in: Central European History, Val. 25, No. 3, S. 251-280.

Ritter, Gerhard, 1979: Vorwort, in: In der Stunde Null. Die Denkschrift des Freiburger BonhoefferKreises, Ttibingen: Mohr (Siebeck), S. 26-30.

Röpke, Wilhelm, 1976: Briefe, 1934-1966. Der innere Kompaß, Erlenbach-Zürich: E. Rentsch.

Rüstow, Alexander, 1932 [1986]: Interessenpolitik oder Staatspolitik?, in: Der Deutsche Volkswirt 6, S. 169 ff. Hier zitiert nach dem Reprint in Wolfram Engels und Hartmut Froels (Hrsg.), 1986: Querschnitte, Düsseldorf: Gesellschaft für Wirtschaftspublizistik, S. 66-71.

Rüstow, Alexander, 1932: Freie Wirtschaft - starker Staat, in: Schriften des Vereins für Socialpolitik, Bd. 187 [hrsg. von Franz Böse: Deutschland und die Weltkrise], München: Duncker \& Humblot, S. 62-69.

Rüstow, Alexander, 1945: Das Versagen des Wirtschaftsliberalismus als religionsgeschichtliches Problem. Istanbuler Schriften 12, Istanbul. $(\leftarrow \mathbf{S . ~ 1 9 7 )}$

Rüstow, Alexander, 1960: Paläoliberalismus, Kollektivismus und Neoliberalismus in der Wirtschafts- und Sozialordnung, in: Christentum und Liberalismus, Studien und Berichte der Katholischen Akademie in Bayern, Heft 13, hrsg. von Karl Forster, S. 149-178.

Rüstow, Alexander, 1963: Rede und Antwort. Hrsg. von Walter Hoch, Ludwigsburg: Martin Hoch.

Sontheimer, Kurt, 1962: Antidemokratisches Denken in der Weimarer Republik: Die politischen Ideen des deutschen Nationalismus zwischen 1918 und 1933, München: Nymphenburger Verlagshandlung.

Stolleis, Michael, 1999: Geschichte des öffentlichen Rechts in Deutschland, Bd. 3: Staats- und Verwaltungsrechtswissenschaft in Republik und Diktatur, 1914-1945, München: Beck.

Thielicke, Helmut, 1979: Zur Erfahrung, in: In der Stunde Null. Die Denkschrift des Freiburger Bonhoeffer-Kreises, Tübingen: Mohr (Siebeck), S. 5-23.

Troeltsch, Ernst, 1923: Die Soziallehren der christlichen Kirchen und Gruppen, Tübingen: Mohr 
(Siebeck). Hier zitiert nach der UTB-Ausgabe von 1994.

vom Bruch, Rüdiger, 1985: Bürgerliche Sozialreform im deutschen Kaiserreich, in:Rüdiger vom

Bruch (Hrsg.), Weder Kommunismus noch Kapitalismus. Bürgerliche Sozialreform in Deutschland vom Vormärz bis zur Ära Adenauer, München: Beck, S. 61-179. 